\title{
Extremal Problems Related to Dual Gauss-John Position
}

\author{
Tongyi Ma \\ College of Mathematics and Statistics, Hexi University, Zhangye, China \\ Email: matongyi@126.com, matongyi_123@163.com
}

How to cite this paper: Ma, T.Y. (2018) Extremal Problems Related to Dual Gauss-John Position. Journal of Applied Mathematics and Physics, 6, 2589-2599.

https://doi.org/10.4236/jamp.2018.612216

Received: December 4, 2018

Accepted: December 23, 2018

Published: December 26, 2018

\begin{abstract}
In this paper, the extremal problem, $\min \left\{\tilde{l}_{p}(\phi K): o \in \phi K \subseteq L, \phi \in \mathrm{GL}(n)\right\}$, of two convex bodies $K$ and $L$ in $\mathbb{R}^{n}$ is considered. For $K$ to be in extremal position in terms of a decomposition of the identity, give necessary conditions together with the optimization theorem of John. Besides, we also consider the weaker optimization problem:

$\min \left\{\left(\tilde{l}_{p}(\phi K)\right)^{p}: \phi K \subseteq B_{2}^{n}, \phi K \cap S^{n-1} \neq \varnothing, \phi \in \mathrm{GL}(n)\right\}$. As an application, we give the geometric distance between the unit ball $B_{2}^{n}$ and a centrally symmetric convex body $K$.

\section{Keywords}

Dual Gauss-John Position, Optimization Theorem of John, Dual $\tilde{l}_{p}$-Norm, Contact Pair
\end{abstract}

\section{Introduction}

Let $\gamma_{n}$ be the classical Gaussian probability measure with density $\frac{1}{(\sqrt{2 \pi})^{n}} e^{-\frac{|x|^{2}}{2}}$, and $\|\cdot\|_{K}$ is the Minkowski functional of a convex body $K \subset \mathbb{R}^{n}$. An important quantity on local theory of Banach space is the associated $l$-norm:

$$
l(K)=\int_{\mathbb{R}^{n}}\|x\|_{K} \mathrm{~d} \gamma_{n}(x) .
$$

The minimum of the functional

$$
\int_{\mathbb{R}^{n}}\|x\|_{\phi K} \mathrm{~d} \gamma_{n}(x)
$$

under the constraint $\phi K \subseteq B_{2}^{n}$ is attained for $\phi=I_{n}$, then a convex body $K$ is in the Gauss-John position, where $\phi \in \mathrm{GL}(n), B_{2}^{n}$ is the Euclidean unit ball 
and $I_{n}$ is the identity mapping from $\mathbb{R}^{n}$ to $\mathbb{R}^{n}$.

For $x \in \mathbb{R}^{n} \backslash\{o\}$, the map $x \otimes x: \mathbb{R}^{n} \rightarrow \mathbb{R}^{n}$ is the rank 1 linear operator $y \mapsto\langle x, y\rangle x$.

Giannopoulos et al. in [1] showed that if $K$ is in the Gauss-John position, then there exist $m \leq n(n+1) / 2$ contact points $x_{1}, x_{2}, \cdots, x_{m} \in \partial K \cap S^{n-1}$, and constants $c_{1}, c_{2}, \cdots, c_{m}>0$ such that $\sum_{i=1}^{m} c_{i}=1$ and

$$
\int_{\mathbb{R}^{n}}\left(x \otimes x-I_{n}\right)\|x\|_{K} \mathrm{~d} \gamma_{n}(x)=\int_{\mathbb{R}^{n}}\|x\|_{K} \mathrm{~d} \gamma_{n}(x)\left(\sum_{i=1}^{m} c_{i} x_{i} \otimes x_{i}\right) .
$$

Note that the Gauss-John position is not equivalent to the classical John position. Giannopoulos et al. [1] pointed out that, when $K$ is in the Gauss-John position, the distance between the unit ball $B_{2}^{n}$ and the John ellipsoid is of order $\sqrt{n / \log n}$.

Notice that the study of the classical John theorem went back to John [2]. It states that each convex body $K$ contains a unique ellipsoid of maximal volume, and when $B_{2}^{n}$ is the maximal ellipsoid in $K$, it can be characterized by points of contact between the boundary of $K$ and that of $B_{2}^{n}$. John's theorem also holds for arbitrary centrally symmetric convex bodies, which was proved by Lewis [3] and Milman [4]. It was provided in [5] that a generalization of John's theorem for the maximal volume position of two arbitrary smooth convex bodies. Bastero and Romance [6] proved another version of John's representation removing smoothness condition but with assumptions of connectedness. For more information about the study of its extensions and applications, please see [7]-[13].

Recall that a convex body $\tilde{K}$ is a position of $K$ if $\tilde{K}=\phi K+a$, for some non-degenerate linear mapping $\phi \in \mathrm{GL}(n)$ and some $a \in \mathbb{R}^{n}$. We say that $K$ is in a position of maximal volume in $L$ if $K \subseteq L$ and for any position $\tilde{K}$ of $K$ such that $\tilde{K} \subseteq L$ we have $\operatorname{vol}_{n}(\tilde{K}) \leq \operatorname{vol}_{n}(K)$, where $\operatorname{vol}_{n}(\cdot)$ denotes the volume of appropriate dimension.

Recently, Li and Leng in [14] generalized the Gauss-John position to a general situation. For $p \geq 1$, denote $l_{p}$-norm by

$$
l_{p}(K)=\left(\int_{\mathbb{R}^{n}}\|x\|_{K}^{p} \mathrm{~d} \gamma_{n}(x)\right)^{\frac{1}{p}} .
$$

They consider the following extremal problem:

$$
\min \left\{l_{p}(\phi K): o \in \phi K \subseteq L, \phi \in \mathrm{GL}(n)\right\},
$$

where $L$ is a given convex body in $\mathbb{R}^{n}$ and $K$ is a convex body containing the origin $o$ such that $o \in K \subseteq L$.

Li and Leng [14] showed that let $L$ be a given convex body in $\mathbb{R}^{n}$ and $K$ be a convex body such that $o \in K \subseteq L$. If $K$ is in extremal position of (1.2), then there exist $m \leq n^{2}$ contact pairs $\left(x_{i}, y_{i}\right)_{1 \leq i \leq m}$ of $(K, L)$, and constants $c_{1}, c_{2}, \cdots, \cdots, c_{m}>0$ such that

$$
I_{n}=\int_{\mathbb{R}^{n}}(x \otimes x) \mathrm{d} \mu(x)-p \sum_{i=1}^{m} c_{i} x_{i} \otimes y_{i}, \quad \sum_{i=1}^{m} c_{i}=1,
$$


where $\mathrm{d} \mu(x)$ is the probability measure on $\mathbb{R}^{n}$ with normalized density

$$
\mathrm{d} \mu(x)=\|x\|_{K}^{p} \mathrm{~d} \gamma_{n}(x) /\left(l_{p}(K)\right)^{p} .
$$

In this paper, we first present a dual concept of $l_{p}$-norm $l_{p}(K)$. The generalizations of John's theorem and Li and Leng [14] play a critical role. It would be impossible to overstate our reliance on their work.

For $p \geq 1$, we define the dual $\tilde{l}_{p}$-norm of convex body $K$ by

$$
\tilde{l}_{p}(K)=\left(\int_{\mathbb{R}^{n}} \rho_{K}(x)^{p} \mathrm{~d} \gamma_{n}(x)\right)^{\frac{1}{p}},
$$

where $\rho_{K}$ is the radial function of the star body $K$ about the origin.

Now, we consider the extremal problem:

$$
\min \left\{\tilde{l}_{p}(\phi K): o \in \phi K \subseteq L, \phi \in \mathrm{GL}(n)\right\},
$$

where $L$ is a given convex body in $\mathbb{R}^{n}$ and $K$ is a convex body containing the origin $o$ such that $o \in K \subseteq L$.

Then we prove that the necessary conditions for $K$ to be in extremal position in terms of a decomposition of the identity.

Theorem 1.1. Let $L$ be a given convex body in $\mathbb{R}^{n}$ and $K$ be a convex body such that $o \in K \subseteq L$. If $K$ is in extremal position of (1.4), then there exist $m \leq n^{2}$ contact pairs $\left(x_{i}, y_{i}\right)_{1 \leq i \leq m}$ of $(K, L)$, and $c_{1}, c_{2}, \cdots, c_{m}>0$ such that

$$
I_{n}=\int_{\mathbb{R}^{n}}(x \otimes x) \mathrm{d} \tilde{\mu}(x)-p \sum_{i=1}^{m} c_{i} x_{i} \otimes y_{i}, \quad \sum_{i=1}^{m} c_{i}=1,
$$

where $\mathrm{d} \tilde{\mu}(x)$ is the probability measure on $\mathbb{R}^{n}$ with normalized density

$$
\mathrm{d} \tilde{\mu}(x)=\|x\|_{K}^{-p} \mathrm{~d} \gamma_{n}(x) /\left(\tilde{l}_{p}(K)\right)^{p} .
$$

Next the following result is obtained, which is an restriction that is weaker than the extremal problem (1.4):

$$
\min \left\{\left(\tilde{l}_{p}(\phi K)\right)^{p}: \phi K \subseteq B_{2}^{n}, \phi K \cap S^{n-1} \neq \varnothing, \phi \in \mathrm{GL}(n)\right\} .
$$

Theoren 1.2. Let $K$ be a given convex body in $\mathbb{R}^{n}$. If $I_{n}$ is the solution of the extremal problem (1.5), then there exist contact points $u, u^{\prime}$ of $K$ and $B_{2}^{n}$ such that

$$
\left\langle u^{\prime}, \theta\right\rangle^{2} \leq\left(\tilde{l}_{p}(K)\right)^{p} \int_{\mathbb{R}^{n}}\|x\|_{K}^{-p-1}\left\langle\nabla h_{K^{o}}(x), \theta\right\rangle\langle x, \theta\rangle d \gamma_{n}(x) \leq\langle u, \theta\rangle^{2},
$$

for every $\theta \in S^{n-1}$.

The rest of this paper is organized as follows: In Section 2, some basic notation and preliminaries are provided. We prove Theorem 1.1 and Theorem 1.2 in Section 3. In particular, as an application of the extremal problem of

$$
\min \left\{\left(\tilde{l}_{p}(\phi K)\right)^{p}: o \in \phi K \subseteq B_{2}^{n}, \phi \in \mathrm{GL}(n)\right\},
$$

Section 3 shows the geometric distance between the unit ball $B_{2}^{n}$ and a centrally symmetric convex body $K$. 


\section{Notation and Preliminaries}

In this section, we present some basic concepts and various facts that are needed in our investigations. We shall work in $\mathbb{R}^{n}$ equipped with the canonical Euclidean scalar product $\langle\cdot, \cdot\rangle$ and write $|\cdot|$ for the corresponding Euclidean norm. We denote the unit sphere by $S^{n-1}$.

Let $K$ be a convex body (compact, convex sets with non-empty interiors) in $\mathbb{R}^{n}$. The support function of $K$ is defined by

$$
h_{K}(x)=\max \{\langle x, y\rangle: y \in K\}, \quad x \in \mathbb{R}^{n} .
$$

Obviously, $h_{\phi K}(x)=h_{K}\left(\phi^{t} x\right)$ for $\phi \in \mathrm{GL}(n)$, where $\phi^{t}$ denotes the transpose of $\phi$.

A set $K \subset \mathbb{R}^{n}$ is said to be a star body about the origin, if the line segment from the origin to any point $x \in K$ is contained in $K$ and $K$ has continuous and positive radial function $\rho_{K}(\cdot)$. Here, the radial function of $K, \rho_{K}: S^{n-1} \rightarrow[0, \infty)$, is defined by

$$
\rho_{K}(u)=\max \{\lambda: \lambda u \in K\} .
$$

Note that if $K$ be a star body (about the origin) in $\mathbb{R}^{n}$, then $K$ can be uniquely determined by its radial function $\rho_{K}(\cdot)$ and vice verse. If $\alpha>0$, we have

$$
\rho_{K}(\alpha x)=\alpha^{-1} \rho_{K}(x) \text { and } \rho_{\alpha K}(x)=\alpha \rho_{K}(x) .
$$

More generally, from the definition of the radial function it follows immediately that for $\phi \in \mathrm{GL}(n)$ the radial function of the image $\phi K=\{\phi y: y \in K\}$ of star body $K$ is given by $\rho_{\phi K}(x)=\rho_{K}\left(\phi^{-1} x\right)$, for all $x \in \mathbb{R}^{n}$.

If $K, L \in \mathcal{S}_{o}^{n}$ and $\lambda, \mu \geq 0$ (not both zero), then for $p>0$, the $L_{p}$-radial combination, $\lambda K \tilde{+}_{p} \mu L \in \mathcal{S}_{o}^{n}$, is defined by (see [15])

$$
\rho\left(\lambda K \tilde{+}_{p} \mu L, \cdot\right)^{p}=\lambda \rho(K, \cdot)^{p}+\mu \rho(L, \cdot)^{p} .
$$

If a star body $K$ contains the origin $o$ as its interior point, then the Minkowski functional $\|\cdot\|_{K}$ of $K$ is defined by

$$
\|x\|_{K}=\min \{\lambda>0: x \in \lambda K\} .
$$

In this case,

$$
\|x\|_{K}=\rho_{K}^{-1}(x)=h_{K^{\circ}}(x),
$$

where $K^{\circ}$ denotes the polar set of $K$, which is defined by

$$
K^{\circ}=\left\{x \in \mathbb{R}^{n}:\langle x, y\rangle \leq 1 \text { for all } y \in K\right\} .
$$

It is easy to verify that for $\phi \in \mathrm{GL}(n)$,

$$
(\phi K)^{\circ}=\phi^{-t} K^{\circ},
$$

where $\phi^{-t}$ denotes the reverse of the transpose of $\phi$. Obviously, $\left(K^{\circ}\right)^{\circ}=K$ (see [13] for details).

Let $K$ and $L$ be two convex bodies in $\mathbb{R}^{n}$. According to [4], if $o \in K \subseteq L \subseteq \mathbb{R}^{n}$, we call a pair $(x, y) \in \mathbb{R}^{n} \times \mathbb{R}^{n}$ a contact pair for $(K, L)$ if it satisfies: 
1) $x \in K \cap \partial L$,

2) $y \in L^{\circ} \cap \partial K^{\circ}$,

3) $\langle x, y\rangle=1$.

If $x, y \in \mathbb{R}^{n}$, we denote by $x \otimes y$ the rank one projection defined by $x \otimes y(u)=\langle x, u\rangle y$ for all $u \in \mathbb{R}^{n}$.

The geometric distance $\delta_{G}(K, L)$ of the convex bodies $K$ and $L$ is defined by

$$
\delta_{G}(K, L)=\inf \{\alpha \beta: \alpha>0, \beta>0,(1 / \beta) L \subset K \subset \alpha L\} .
$$

\section{Proof of Main Results}

First, we prove that $\tilde{l}_{p}(\cdot)$ is a norm with respect to $L_{p}$-radial combination in $\mathcal{S}_{o}^{n}$. Apparently, $\tilde{l}_{p}(K) \geq 0$ and $\tilde{l}_{p}(K)=0$ if and only if $K=\{o\}$. At the same time, $\tilde{l}_{p}(c K)=c \tilde{l}_{p}(K)$ if real constant $c>0$. In addition, it is follows that

$$
\tilde{l}_{p}\left(K \tilde{+}_{p} L\right) \leq \tilde{l}_{p}(K)+\tilde{l}_{p}(L)
$$

Indeed, we have

$$
\begin{aligned}
& \tilde{l}_{p}\left(K \tilde{+}_{p} L\right)=\left(\int_{\mathbb{R}^{n}} \rho_{K \tilde{p}_{p} L}^{p}(x) \mathrm{d} \gamma_{n}(x)\right)^{\frac{1}{p}} \\
& =\left(\int_{\mathbb{R}^{n}} \rho_{K}^{p}(x) \mathrm{d} \gamma_{n}(x)+\int_{\mathbb{R}^{n}} \rho_{L}^{p}(x) \mathrm{d} \gamma_{n}(x)\right)^{\frac{1}{p}} \\
& \leq\left(\int_{\mathbb{R}^{n}} \rho_{K}^{p}(x) \mathrm{d} \gamma_{n}(x)\right)^{\frac{1}{p}}+\left(\int_{\mathbb{R}^{n}} \rho_{L}^{p}(x) \mathrm{d} \gamma_{n}(x)\right)^{\frac{1}{p}} \\
& =\tilde{l}_{p}(K)+\tilde{l}_{p}(L) .
\end{aligned}
$$

Therefore, $\tilde{l}_{p}(\cdot)$ is a norm with respect to $L_{p}$-radial combination and $\mathcal{S}_{o}^{n}$ is normed space for $\tilde{l}_{p}(\cdot)$.

Now, we prove the optimization theorem of John [2] (see [10] also).

Lemma 3.1. Let $\mathcal{F}: \mathbb{R}^{N} \rightarrow \mathbb{R}$ be a $C^{1}$-function. Let $S$ be a compact metric space and $\mathcal{G}: \mathbb{R}^{N} \times S \rightarrow \mathbb{R}$ be continuous. Suppose that for every $s \in S$, $\nabla_{z} \mathcal{G}(z, s)$ exists and is continuous on $\mathbb{R}^{N} \times S$.

Let $\mathcal{A}=\left\{z \in \mathbb{R}^{N}: \mathcal{G}(z, s) \geq 0\right.$, for all $\left.s \in S\right\}$ and $z_{0} \in \mathcal{A}$ satisfy

$$
\mathcal{F}\left(z_{0}\right)=\min _{z \in \mathcal{A}} \mathcal{F}(z) \text {. }
$$

Then, either $\nabla_{z} \mathcal{F}\left(z_{0}\right)=0$, or, for some $1 \leq m \leq N$, there exist $s_{1}, s_{2}, \cdots, s_{m} \in S$ and $\lambda_{1}, \lambda_{2}, \cdots, \lambda_{m} \in \mathbb{R}$ such that $\mathcal{G}\left(z_{0}, s_{i}\right)=0, \lambda_{i} \geq 0$ for $1 \leq i \leq m$, and

$$
\nabla_{z} \mathcal{F}\left(z_{0}\right)=\sum_{i=1}^{m} \lambda_{i} \nabla_{z} \mathcal{G}\left(z_{0}, s_{i}\right)
$$

Using a similar argument as that in [1], we give the proof of Theorem 1.1.

Proof of Theorem 1.1. For $N=n^{2}$, we define $\mathcal{F}: \mathbb{R}^{N} \rightarrow \mathbb{R}$ by

$$
\mathcal{F}(\phi)=\tilde{l}_{p}(\phi K)=\left(\int_{\mathbb{R}^{n}}\left\|\phi^{-1} x\right\|_{K}^{-p} \mathrm{~d} \gamma_{n}(x)\right)^{\frac{1}{p}},
$$

where $\phi \in \mathbb{R}^{N}$ is the linear mapping from $\mathbb{R}^{n}$ to $\mathbb{R}^{n}$. Clearly $\mathcal{F}$ is $C^{1}$. For $S=K \times L^{\circ}$, define $\mathcal{G}: \mathbb{R}^{N} \times S \rightarrow \mathbb{R}$ by 


$$
\mathcal{G}(\phi,(x, y))=1-\langle\phi x, y\rangle
$$

The set

$$
\mathcal{A}=\left\{z \in \mathbb{R}^{N}: \mathcal{G}(z, s) \geq 0, s \in S\right\}
$$

is just the set of elements $\phi \in \mathbb{R}^{N}$ such that $\phi K \subseteq L$. If $K$ is in extremal position of $\min \left\{\tilde{l}_{p}(\phi K): o \in \phi K \subseteq L, \phi \in \mathrm{GL}(n)\right\}$, then $\mathcal{F}$ attains its minimum on $\mathcal{A}$ at $I_{n}$, namely,

$$
\mathcal{F}\left(I_{n}\right)=\tilde{l}_{p}(K)=\min \left\{\tilde{l}_{p}(\phi K): o \in \phi K \subseteq L, \phi \in \mathrm{GL}(n)\right\} .
$$

Now we prove $\nabla_{\phi} \mathcal{F}\left(I_{n}\right)$. It follows from (3.1) that

$$
\begin{aligned}
\mathcal{F}(\phi) & =\left(\int_{\mathbb{R}^{n}}\left\|\phi^{-1} x\right\|_{K}^{-p} \mathrm{~d} \gamma_{n}(x)\right)^{\frac{1}{p}} \\
& =\left((2 \pi)^{-\frac{n}{2}} \int_{\mathbb{R}^{n}}\left\|\phi^{-1} x\right\|_{K}^{-p} e^{-\frac{|x|^{2}}{2}} \mathrm{~d} x\right)^{\frac{1}{p}} \\
& =\left((2 \pi)^{-\frac{n}{2}}(\operatorname{det} \phi) \int_{\mathbb{R}^{n}}\|x\|_{K}^{-p} e^{-\frac{|\phi x|^{2}}{2}} \mathrm{~d} x\right)^{\frac{1}{p}} .
\end{aligned}
$$

It is easy to obtain that for non-degenerate $\phi$, we have

$$
\nabla_{\phi} \mathcal{G}(\phi,(x, y))=-\nabla_{\phi}\langle\phi x, y\rangle=\nabla_{\phi}\langle x \otimes y, \phi\rangle=-x \otimes y
$$

and

$$
\begin{aligned}
\nabla_{\phi} \mathcal{F}(\phi)= & \frac{1}{p}\left((2 \pi)^{-\frac{n}{2}}(\operatorname{det} \phi) \int_{\mathbb{R}^{n}}\|x\|_{K}^{-p} e^{-\frac{|\phi x|^{2}}{x}} \mathrm{~d} x\right)^{-\frac{1}{q}} \\
& \times\left[(2 \pi)^{-\frac{n}{2}}(\operatorname{det} \phi)\left(\phi^{-1}\right)^{*} \int_{\mathbb{R}^{R^{n}}}\|x\|_{K}^{-p} e^{-\frac{|\phi x|^{2}}{x}} \mathrm{~d} x\right. \\
& \left.-(2 \pi)^{-\frac{n}{2}}(\operatorname{det} \phi) \int_{\mathbb{R}^{n}}\|x\|_{K}^{-p} e^{-\frac{|\phi x|^{2}}{x}} x \otimes x \mathrm{~d} x\right],
\end{aligned}
$$

where $\frac{1}{p}+\frac{1}{q}=1,\left(\phi^{-1}\right)^{*}$ denotes conjugate of transposed transformation of $\phi^{-1}$, and $\phi^{-1}$ is inverse transform of $\phi \in \mathrm{GL}(n)$.

Since $\mathcal{F}$ attains its minimum on $\mathcal{A}$ at $z_{0}=I_{n}$, combining with Lemma 3.1 , it follows that for some $m \leq N$, there exist $\lambda_{i} \geq 0, s_{i} \in S, s_{i}=\left(x_{i}, y_{i}\right)$, $1 \leq i \leq m$, such that

$$
\left\langle x_{i}, y_{i}\right\rangle=1-\mathcal{G}\left(I_{n},\left(x_{i}, y_{i}\right)\right)=1,1 \leq i \leq m,
$$

and

$$
\begin{aligned}
\nabla_{\phi} \mathcal{F}\left(I_{n}\right) & =\frac{1}{p}\left(\tilde{l}_{p}(K)\right)^{-\frac{p}{q}} \int_{\mathbb{R}^{n}}\left(I_{n}-x \otimes x\right)\|x\|_{K}^{-p} \mathrm{~d} \gamma_{n}(x) \\
& =\sum_{i=1}^{m} \lambda_{i} \nabla_{\phi} \mathcal{G}\left(I_{n},\left(x_{i}, y_{i}\right)\right) \\
& =-\sum_{i=1}^{m} \lambda_{i} x_{i} \otimes y_{i} .
\end{aligned}
$$


From $\left\langle x_{i}, y_{i}\right\rangle=1, x_{i} \in K \subseteq L, y_{i} \in L^{\circ} \subseteq K^{\circ}$, we yield $x_{i} \in \partial L$ and $y_{i} \in \partial K^{\circ}$. Taking the trace in (3.2), we have

$$
\begin{aligned}
\operatorname{Tr} & \left(\nabla_{\phi} \mathcal{F}\left(I_{n}\right)\right) \\
& =\operatorname{Tr}\left(\frac{1}{p}\left(\tilde{l}_{p}(K)\right)^{-\frac{p}{q}} \int_{\mathbb{R}^{n}}\left(I_{n}-x \otimes x\right)\|x\|_{K}^{-p} \mathrm{~d} \gamma_{n}(x)\right) \\
& =\frac{1}{p}\left(\tilde{l}_{p}(K)\right)^{-\frac{p}{q}}\left[n \int_{\mathbb{R}^{n}}\|x\|_{K}^{-p} \mathrm{~d} \gamma_{n}(x)-\int_{\mathbb{R}^{n}}|x|^{2}\|x\|_{K}^{-p} \mathrm{~d} \gamma_{n}(x)\right] \\
& =\frac{1}{p}\left(\tilde{l}_{p}(K)\right)^{-\frac{p}{q}}\left[n \int_{0}^{\infty} r^{n-p-1} e^{-\frac{r^{2}}{2}} \mathrm{~d} r-\int_{0}^{\infty} r^{n-p+1} e^{-\frac{r^{2}}{2}} \mathrm{~d} r\right] \int_{S^{n-1}}\|\theta\|_{K}^{-p} \mathrm{~d} S(\theta) \\
& =\frac{1}{p}\left(\tilde{l}_{p}(K)\right)^{-\frac{p}{q}}\left(p \int_{\mathbb{R}^{n}}\|x\|_{K}^{-p} \mathrm{~d} \gamma_{n}(x)\right)=\tilde{l}_{p}(K) .
\end{aligned}
$$

Suppose $\lambda_{i}=c_{i} \tilde{l}_{p}(K)$. Together with (3.2), we obtain

$$
\int_{\mathbb{R}^{n}}\left(x \otimes x-I_{n}\right)\|x\|_{K}^{-p} \mathrm{~d} \gamma_{n}(x)=p\left(\tilde{l}_{p}(K)\right)^{p}\left(\sum_{i=1}^{m} c_{i} x_{i} \otimes y_{i}\right),
$$

where $\sum_{i=1}^{m} c_{i}=1$. This completes the proof.

If $L=B_{2}^{n}$ and $\mathcal{G}(\phi, x)=1-|\phi x|^{2}$, then using the same method in the proof of Theorem 1.1, we obtain

Corollary 3.2. Let $K$ be a convex body such that $o \in K \subseteq B_{2}^{n}$. If $K$ is in extremal position of (1.7), then there exist contact points $u_{1}, u_{2}, \cdots, u_{m} \in \partial K \cap S^{n-1}$ with $m \leq n^{2}$ and $c_{1}, c_{2}, \cdots, c_{m}>0$, such that,

$$
I_{n}=\int_{\mathbb{R}^{n}}(x \otimes x) \mathrm{d} \tilde{\mu}(x)-p \sum_{i=1}^{m} c_{i} u_{i} \otimes u_{i}, \quad \sum_{i=1}^{m} c_{i}=1,
$$

where $\mathrm{d} \tilde{\mu}(x)$ is the probability measure on $\mathbb{R}^{n}$ with normalized density

$$
\mathrm{d} \tilde{\mu}(x)=\|x\|_{K}^{-p} \mathrm{~d} \gamma_{n}(x) /\left(\tilde{l}_{p}(K)\right)^{p} .
$$

Proof of Theorem 1.2. Suppose that $\phi \in L\left(\mathbb{R}^{n}, \mathbb{R}^{n}\right)$ and $\varepsilon>0$ is small enough. Then

$$
\phi_{1}:=\left(\min _{u \in S^{n-1}}\|u-\varepsilon \phi u\|_{K}\right)\left(I_{n}-\varepsilon \phi\right)^{-1}
$$

satisfies $\phi_{1} K \subseteq B_{2}^{n}, \phi_{1} K \cap S^{n-1} \neq \varnothing$. Therefore

$$
\int_{\mathbb{R}^{n}}\|x-\varepsilon \phi x\|_{K}^{-p} \mathrm{~d} \gamma_{n}(x) \leq\left(\tilde{l}_{p}(K)\right)^{p}\left(\min _{u \in S^{n-1}}\|u-\varepsilon \phi u\|_{K}\right)^{-p} .
$$

Let $u_{\varepsilon}$ be a point on $S^{n-1}$ at which the minimum is attained. Observe that

$$
\|x-\varepsilon \phi x\|_{K}^{-p}=\|x\|_{K}^{-p}+\varepsilon p\|x\|_{K}^{-p-1}\left\langle\nabla h_{K^{\circ}}(x), \phi x\right\rangle+O\left(\varepsilon^{2}\right)
$$

and

$$
\left|u_{\varepsilon}-\varepsilon \phi u_{\varepsilon}\right|^{-p}=1+\varepsilon p\left\langle u_{\varepsilon}, \phi u_{\varepsilon}\right\rangle+O\left(\varepsilon^{2}\right) .
$$

Since $u_{\varepsilon} \in S^{n-1}$ and $\|\cdot\|_{K} \geq|\cdot|$, we have 


$$
\begin{array}{rl}
\int_{\mathbb{R}^{n}} & p\|x\|_{K}^{-p-1}\left\langle\nabla h_{K^{\circ}}(x), \phi x\right\rangle \mathrm{d} \gamma_{n}(x)+O(\varepsilon) \\
& \leq\left(\tilde{l}_{p}(K)\right)^{p} \frac{\left(\min _{u \in S^{n-1}}\|u-\varepsilon \phi u\|_{K}\right)^{-p}-1}{\varepsilon} \\
& \leq\left(\tilde{l}_{p}(K)\right)^{p} \frac{\left|u_{\varepsilon}-\varepsilon \phi u_{\varepsilon}\right|^{-p}-1}{\varepsilon} \\
& =\left(\tilde{l}_{p}(K)\right)^{p}\left(p\left\langle u_{\varepsilon}, \phi u_{\varepsilon}\right\rangle+O(\varepsilon)\right) .
\end{array}
$$

If $u$ is a contact point of $K$ and $B_{2}^{n}$, then

$$
1+\varepsilon\|\phi\| \geq\|u-\varepsilon \phi u\|_{K} \geq\left\|u_{\varepsilon}-\varepsilon \phi u_{\varepsilon}\right\|_{K} \geq\left\|u_{\varepsilon}\right\|_{K}-\varepsilon\|\phi\| .
$$

It follows that

$$
1 \leq\left\|u_{\varepsilon}\right\|_{K} \leq 1+2 \varepsilon\|\phi\| \text {. }
$$

In order to obtain a sequence $\varepsilon_{k} \rightarrow 0$ and a point $u \in S^{n-1}$ such that $u_{\varepsilon_{k}} \rightarrow u$. If $k \rightarrow \infty$, it follows from (3.4) that $\|u\|_{K}=\lim _{k \rightarrow \infty}\left\|u_{\varepsilon_{k}}\right\|=1$. Namely, $u$ is a contain point of $K$ and $B_{2}^{n}$. By (3.3), we obtain

$$
\int_{\mathbb{R}^{n}}\|x\|_{K}^{-p-1}\left\langle\nabla h_{K^{\circ}}(x), \phi x\right\rangle \mathrm{d} \gamma_{n}(x) \leq\left(\tilde{l}_{p}(K)\right)^{p}\langle u, \phi u\rangle .
$$

Taking $\phi$ for $-\phi$, we can find another contact point $u^{\prime}$ of $K$ and $B_{2}^{n}$ such that

$$
\int_{\mathbb{R}^{n}}\|x\|_{K}^{-p-1}\left\langle\nabla h_{K^{\circ}}(x), \phi x\right\rangle \mathrm{d} \gamma_{n}(x) \geq\left(\tilde{l}_{p}(K)\right)^{p}\left\langle u^{\prime}, \phi u^{\prime}\right\rangle .
$$

Choosing $\phi_{\theta}(x)=\langle x, \theta\rangle \theta$ with $\theta \in S^{n-1}$, we get (1.6).

\section{Estimate of the Distance}

Lemma 4.1. (see [16]) Let $x=\left(x_{1}, x_{2}, \cdots, x_{n}\right) \in \mathbb{R}^{n}$ and

$$
\begin{aligned}
y=\left(y_{1}, y_{2}, \cdots, y_{n}\right) & \in \mathbb{R}^{n} . \text { If } \\
0 & <m_{1} \leq x_{k} \leq M_{1}, 0<m_{2} \leq y_{k} \leq M_{2}, k=1, \cdots, n,
\end{aligned}
$$

then

$$
\left(\sum_{k=1}^{n} x_{k}^{2}\right)\left(\sum_{k=1}^{n} y_{k}^{2}\right) \leq\left(\frac{\sqrt{\frac{M_{1} M_{2}}{m_{1} m_{2}}}+\sqrt{\frac{m_{1} m_{2}}{M_{1} M_{2}}}}{2}\right)^{2}\left(\sum_{k=1}^{n} x_{k} y_{k}\right)^{2} .
$$

Lemma 4.1 implies that if $x, y \in \mathbb{R}^{n}$, then there exist a constant $c \in(0,1)$ such that

$$
|\langle x, y\rangle| \geq c|x||y| .
$$

Suppose that $K$ is a centrally symmetric convex body in $\mathbb{R}^{n}$ such that $K$ is in the extremal position of (1.7). Now we estimate the geometric distance between $K$ and $B_{2}^{n}$.

Theorem 4.1. Let $K \subseteq B_{2}^{n}$ be a centrally symmetric convex body in $\mathbb{R}^{n}$. If $K$ is in the extremal position of (1.7) and $1 \leq p<3$, then

$$
\tilde{c}_{n, p} B_{2}^{n} \subseteq K \subseteq B_{2}^{n} \text {, }
$$


where

$$
\tilde{c}_{n, p}=\frac{\tilde{l}_{p}\left(B_{2}^{n}\right)}{\sqrt{n}}\left(\frac{\sqrt{\pi}(c p+1)}{2^{1-\frac{p}{2}} \Gamma\left(\frac{3-p}{2}\right)}\right)^{\frac{1}{p}}, c \in(0,1) .
$$

Proof. It follows from Corollary 3.2 that $K$ satisfies

$$
I_{n}=\int_{\mathbb{R}^{n}}(x \otimes x) \mathrm{d} \tilde{\mu}(x)-p \sum_{i=1}^{m} c_{i} u_{i} \otimes u_{i}, \quad \sum_{i=1}^{m} c_{i}=1,
$$

where $\mathrm{d} \tilde{\mu}(x)$ is the probability measure on $\mathbb{R}^{n}$ with normalized density

$$
\mathrm{d} \tilde{\mu}(x)=\|x\|_{K}^{-p} \mathrm{~d} \gamma_{n}(x) /\left(\tilde{l}_{p}(K)\right)^{p} .
$$

For $y \in K^{\circ}$ and $u_{i} \in S^{n-1}$. By (4.1), there exists a constant $c \in(0,1)$ such that $\left|\left\langle y, u_{i}\right\rangle\right| \geq c|y|$. So we obtain

$$
\int_{\mathbb{R}^{n}}\left(|\langle x, y\rangle|^{2}-|y|^{2}\right) \mathrm{d} \tilde{\mu}(x) \geq c p|y|^{2} \sum_{i=1}^{m} c_{i}=c p|y|^{2} .
$$

That is,

$$
(c p+1)|y|^{2} \leq \int_{\mathbb{R}^{n}}|\langle x, y\rangle|^{2} \mathrm{~d} \tilde{\mu}(x) .
$$

Since $\|x\|_{K} \geq|\langle x, y\rangle|$, we have

$$
\begin{aligned}
\int_{\mathbb{R}^{n}}|\langle x, y\rangle|^{2}\|x\|_{K}^{-p} \mathrm{~d} \gamma_{n}(x) & \leq \int_{\mathbb{R}^{n}}|\langle x, y\rangle|^{2-p} \mathrm{~d} \gamma_{n}(x) \\
& =(2 \pi)^{-\frac{n}{2}} \int_{S^{n-1}}|\langle\theta, y\rangle|^{2-p} \mathrm{~d} S(\theta) \int_{0}^{\infty} r^{n-p+1} e^{-\frac{r^{2}}{2}} \mathrm{~d} r \\
& =2^{1-\frac{p}{2}} \pi^{-\frac{1}{2}} \Gamma\left(\frac{3-p}{2}\right)|y|^{2-p} .
\end{aligned}
$$

From John's theorem, for every centrally symmetric convex body $K$ in $\mathbb{R}^{n}$, there is a corresponding to the ball $\lambda B_{2}^{n}$ such that $\lambda B_{2}^{n} \subseteq K \subseteq \sqrt{n} \lambda B_{2}^{n}(\lambda>0)$. Take $\lambda=1 / \sqrt{n}$. We obtain $\frac{1}{\sqrt{n}} B_{2}^{n} \subseteq K \subseteq B_{2}^{n}$. Thus,

$$
\frac{1}{\sqrt{n}} \tilde{l}_{p}\left(B_{2}^{n}\right) \leq \tilde{l}_{p}(K) \leq \tilde{l}_{p}\left(B_{2}^{n}\right) \text {. }
$$

Therefore, we get

$$
|y| \leq \frac{\sqrt{n}}{\tilde{l}_{p}\left(B_{2}^{n}\right)}\left(\frac{2^{1-\frac{p}{2}} \Gamma\left(\frac{3-p}{2}\right)}{\sqrt{\pi}(c p+1)}\right)^{\frac{1}{p}},
$$

and the result yields.

Giannopoulos et al. in [5] proved that if $K$ is in a position of maximal volume in $L$, then $K \subseteq L \subseteq n K$, which is equivalent to $\frac{1}{n}\|x\|_{K} \leq\|x\|_{L} \leq\|x\|_{K}$ for all $x \in \mathbb{R}^{n}$. Hence it follows that 


$$
1 \leq \frac{\tilde{l}_{p}(L)}{\tilde{l}_{p}(K)} \leq n .
$$

Furthermore, let $\phi \in \mathrm{GL}(n)$. Since $\phi K \subseteq B_{2}^{n}$ is in the maximal volume position of $K$ contained in $B_{2}^{n}$, we have $\frac{1}{\sqrt{n}} B_{2}^{n} \subseteq \phi K \subseteq B_{2}^{n}$. Thus

$$
\frac{1}{\sqrt{n}} \leq \frac{\tilde{l}_{p}(\phi K)}{\tilde{l}_{p}\left(B_{2}^{n}\right)} \leq 1 .
$$

Finally, we propose the following concept of $l_{0}$-norm: Let $K$ be a convex body in $\mathbb{R}^{n}$, we define $l_{0}$-norm by

$$
l_{0}(K)=\exp \left(\int_{\mathbb{R}^{n}} \log \|x\|_{K} \gamma_{n}(x)\right)
$$

We propose an open question as follows: How should we solve the extreme problem

$$
\min \left\{l_{0}(\phi K): o \in \phi K \subseteq L, \phi \in \mathrm{GL}(n)\right\} ?
$$

\section{Funding}

This work is supported by the National Natural Science Foundation of China (Grant No.11561020).

\section{Conflicts of Interest}

The author declares no conflicts of interest regarding the publication of this paper.

\section{References}

[1] Giannopoulos, A., Milman, V. and Rudelson, M. (2000) Convex Bodies with Minimal Mean Width. Geometric Aspects of Functional Analysis, Lecture Notes in Mathematics, 1745, Springer, Berlin, 81-93.

[2] John, F. (1948) Extremum Problems with Inequalities as Subsidiary Conditions. Studies and Essays Presented to R. Courant on His 60th Birthday, January 8, 1948, Interscience Publishers, Inc., New York, 187-204.

[3] Lewis, D. (1979) Ellipsoids Defined by Banach Ideal Norms. Mathematika, 26, 18-29. https://doi.org/10.1112/S0025579300009566

[4] Tomczak-Jaegermann, N. (1989) Banach-Mazur Distances and Finite-Dimensional Operator Ideals. Pitman Monographs and Surveys in Pure and Applied Mathematics, 38, Pitman, London.

[5] Giannopoulos, A., Perissinaki, I. and Tsolomitis, A. (2001) Johns Theorem for an Arbitrary Pair of Convexbodies. Geom. Dedicata, 84, 63-79. https://doi.org/10.1023/A:1010327006555

[6] Bastero and Romance, M. (2002) Johns Decomposition of the Identity in the Non-Convex Case. Positivity, 6, 1-16. https://doi.org/10.1023/A:1012087231191

[7] Ball, K. (1997) An Elementary Introduction to Modern Convex Geometry. In: Flavors of Geometry, Math. Sci.Res. Inst. Publ., 31, Cambridge University Press, Cambridge. 
[8] Bastero, J., Bernues, J. and Romance, M. (2007) From John to Gauss-John Positions via Dual Mixed Volumes. J. Math. Anal. Appl., 328, 550-566.

https://doi.org/10.1016/j.jmaa.2006.05.047

[9] Giannopoulos, A. and Milman, V.D. (2000) Extremal Problems and Isotropic Positions of Convex Bodies. Israel J. Math., 117, 29-60.

https://doi.org/10.1007/BF02773562

[10] Gordon, Y., Litvak, A.E., Meyer, M., et al. (2004) Johns Decomposition in the General Case and Applications. J. Differential Geom., 68, 99-119. https://doi.org/10.4310/jdg/1102536711

[11] Li, A.J., Wang, G. and Leng, G. (2011) An Extended Loomis-Whitney Inequality for Positive Double John Bases. Glasg. Math. J., 53, 451-462.

https://doi.org/10.1017/S0017089511000061

[12] Li, A.J. and Leng, G. (2011) Brascamp-Lieb Inequality for Positive Double John Basis and Its Reverse. Sci. China Math., 54, 399-410. https://doi.org/10.1007/s11425-010-4093-5

[13] Pisier, G. (1989) The Volume of Convex Bodies and Banach Space Geometry. Cambridge Tracts in Mathematics, 94, Cambridge University Press, Cambridge. https://doi.org/10.1017/CBO9780511662454

[14] Li, A.J. and Leng, G.S. (2012) Extremal Problems Related to Gauss-John Position. Acta Mathematica Sinica, English Series, 28, 2527-2534. https://doi.org/10.1007/s10114-012-9735-9

[15] Gardner, R.J. (2002) The Brunn-Minkowski Inequality. Bull. Amer. Math. Soc., 39, 355-405. https://doi.org/10.1090/S0273-0979-02-00941-2

[16] Pólya, G. and Szegö, G. (1925) Aufgaben und Lehrsãtzeaus der Analysis. Vol. 1, Berlin, p. 57, 213-214. 